\title{
Simplified Solar Modulation Model of Inner Trapped Belt Proton Flux As a Function of Atmospheric Density
}

\author{
M. A. K. Lodhi ${ }^{*}$ and Abel B. Diaz \\ Department of Physics, Texas Tech University, MS 1051 Lubbock, TX 79409, USA \\ Thomas L. Wilson \\ NASA, Johnson Space Center, Houston, TX 77058, USA
}

\begin{abstract}
No simple algorithm seems to exist for calculating proton fluxes and lifetimes in the Earth's inner, trapped radiation belt throughout the solar cycle. Most models of the inner trapped belt in use depend upon AP8 which only describes the radiation environment at solar maximum and solar minimum in Cycle 20. One exception is NOAAPRO which incorporates flight data from the TIROS/NOAA polar orbiting spacecraft. The present study discloses yet another, simple formulation for approximating proton fluxes at any time in a given solar cycle, in particular between solar maximum and solar minimum. It is derived from AP8 using a regression algorithm technique from nuclear physics. From flux and its time integral fluence, one can then approximate dose rate and its time integral dose. It has already been published in this journal that the absorbed dose rate, $D$, in the trapped belts exhibits a power law relationship, $D=A \rho^{-n}$, where $A$ is a constant, $\rho$ is the atmospheric density, and the index $n$ is weakly dependent upon shielding. However, that method does not work for flux and

\footnotetext{
${ }^{*}$ Corresponding author. Tel.: +1-806-742-3778; fax: +1-806-742-1182.

E-mail address: a.lodhi@ttu.edu (M.A.K. Lodhi).
} 
fluence. Instead, we extend this idea by showing that the power law approximation for flux $J$ is actually bivariant in energy $E$ as well as density $\rho$. The resulting relation is $J(E, \rho) \sim \sum A\left(E^{n}\right) \rho^{-n}$, with $A$ itself a power law in $E$. This provides another method for calculating approximate proton flux and lifetime at any time in the solar cycle. These in turn can be used to predict the associated dose and dose rate.

\section{Introduction}

Studies of space radiation and its effects are concerned with the impact of charged species on the functionality and lifetime of human beings as well as scientific instrumentation and advanced electronic systems in space. Two aspects of the near-Earth space environment are very relevant, particularly in the thermosphere $(85 \mathrm{~km}<h<500 \mathrm{~km})$ where Shuttle and International Space Station (ISS) orbits occur. One is the existence of energetic proton and electron populations trapped by the Earth's magnetic field in "Van Allen" belts (E.g., Schulz and Lanzerotti, 1974; Spejeldvik and Rothwell, 1985). The other is the realization (Jacchia, 1960, 1961) that the properties of the upper atmosphere of the Earth are strongly coupled to solar activity, in particular atmospheric density and temperature. Throughout the course of the solar cycle, the Earth's atmospheric neutrals expand and contract the thermosphere in response to the behavior of the Sun. Clearly, the density in Jacchia's concept of a dynamic atmosphere couples to the charged-belt species as these undergo multiple scattering off the neutrals. That in turn reduces their 
lifetime in the belts (Blanchard and Hess, 1964; Cornwell et al., 1965; Dragt, 1966; Ray, 1966; Kern, 1994; Pfitzer, 1989; Watts et al., 1989).

Therefore, it becomes necessary to understand how atmospheric density per se couples to charged-belt population levels as a function of solar activity. This is the simplified goal of the present investigation.

Pfitzer (1989; 1990) has succeeded in developing a reasonable parametric method for estimating dose in the thermosphere from atmospheric density. However, the method does not work for flux. Inspired by that preliminary investigation, Badhwar and his colleagues (1999, 1997, 1996a,b; Golightly et al., 1996) have examined flight data for a correlation between dose and atmospheric density. They have extensively studied and analyzed the low-Earth radiation and time lag of the twenty-two year solar modulation of the trapped proton radiation exposure inside the Space Shuttle. They have shown that the daily trapped-particle dose rate is an approximate power law function of daily atmospheric density, thus supporting the Pfitzer model and method. Their further analysis of the trapped absorbed dose rate, $D$, at six fixed locations in the habitable volume of the Shuttle exhibits a power law relationship, $D=A \rho^{-n}$, where $\rho$ is the atmospheric density. The index, $n$, is weakly dependent on the shielding, decreasing as the average shielding increases (Badhwar, 1999).

This present study further examines the AP8 proton flux question and its relationship to atmospheric density. It enhances the previous Pfitzer and Badhwar density analyses by developing a dynamic trapped-belt proton radiation algorithm that is applicable to the ISS and other space flights in the Earth's thermosphere throughout the 
solar cycle. Although only a very limited range of energies is considered, the method addresses several of the shortcomings and over-simplifications in that earlier work.

\section{Analysis}

The limitations with the original NASA trapped-belt models (Sawyer and Vette, 1976; Bilitza, 1987) known as AP8 and AE8 have been thoroughly discussed (Watts et al., 1989; Pfitzer, 1989, 1990; Badhwar, 1999). The AP8 model was constructed from satellite data in solar cycle 20, a small one compared to more recent events. AP8MIN derives from the epoch of 1964, and AP8MAX from that of 1970. The solar radio flux at $10.7 \mathrm{~cm}, F_{10.7}$, is 150 for AP8MAX and 70 for AP8MIN. These baseline values will be adopted here.

One other promising approach to overcoming the AP8 model limitations has already been produced. It involved the development of a new computer technique known as NOAAPRO (Huston and Pfitzer, 1998a,b). This method has since been adapted by Singleterry et al. (2004) to enhance the out-of-date AP8 and AE8 models at Shuttle and ISS altitudes using the computer program SIREST.

Since the original AP8 model is readily available elsewhere (Heynderickx et al., 2004), it will be used to modify the atmospheric density method of Pfitzer and Badhwar by producing a bivariant energy-density algorithm and then compare the result with the NOAAPRO-enhanced AP8 model of Singleterry et al. At the outset, AP8 is adopted here primarily in order to be consistent with the Pfitzer method. The analysis can be applied 
to other simulation methods such as NOAAPRO and SIREST. Only the omnidirectional fluxes are studied in this analysis, noting that the anisotropic nature of these has been discussed by Watts et al. (1989).

Upon examining the proton flux data from the AP8 model program, and in view of the overall problem as studied for more than 40 years (Pfitzer, 1989, 1990), several observations can be summarized.

1. The atmospheric density decreases rapidly as the altitude increases.

2. Both integrated and differential flux for protons increases as the altitude increases (or density decreases) for both solar maximum and minimum cycles.

3. The fluxes in general are smaller at solar max than at solar min, at least for low altitudes and low energies.

4. However, the difference in the proton flux is wider at low energy than for the higher-energy protons at the same altitude.

5. The flux decreases as the energy increases.

These observations prompt the idea that the proton flux $J$ can be expressed, empirically at least, as a function of two variables, density (altitude) and energy, namely $J(E, \rho)$ or $J(E, h)$.

Solar Modulation of Atmospheric Density

The altitude and density relationship has a long history (Jacchia, 1960, 1961; Harris and Priester, 1962, 1963). The form to be used here has been described recently 
by several authors (Pfitzer, 1989, 1990; Watts et al., 1989). A simple parameterization of the US Air Force Model made by Pfitzer $(1989,1990)$ is

$$
\rho=\rho_{o} \exp \left\{-(h-120) /\left[M(h-103)^{1 / 2}\right]\right\}
$$

where the solar-cycle modulation term $M$ is expressed as

$$
\begin{aligned}
& M=0.99+0.518\left\{\left(F_{10.7}+F_{b a r}\right) / 110\right\}^{1 / 2}, \\
& F=F_{10.7}+F_{b a r} .
\end{aligned}
$$

In (1) $\rho$ denotes the atmospheric density, $\rho_{o}$ is assumed to be $\rho_{o}=2.7 \times 10^{-11} \mathrm{~g} / \mathrm{cm}^{3}, h$ is the altitude in $\mathrm{km}, F_{10.7}$ is the instantaneous value of the solar radio flux at $10.7 \mathrm{~cm}$, and $F_{b a r}$ is the weighted value of $F_{10.7}$ for averaging, such as three prior solar rotations.

The density in (1) is a multi-variant function of $h$ and $F_{10.7}$. Similarly, the AP8 proton flux $J$ is a multi-variant function of $h$ and energy $E$. The problem at hand, then, is to generate the multi-dimensional surface of $J$ as a function of $E$ and $h$ or $\rho$. By selecting an altitude $h$ and emulating the solar cycle with a solar radio flux $F_{10.7}$, the modulated proton flux $J$ follows as a function of energy $E$. Dynamically speaking, furthermore, all of these variables are functions of time $t$.

A “carpet" plot (in the sense of Pfitzer, 1989, 1990) is merely a projection of these surfaces onto a two-dimensional graph. This can be obtained for the solar-cycle terms altitude $h=f(\rho)$ and flux $F_{10.7}=g(\rho)$ as a function of atmospheric density $\rho$ in (1) 
and (2), by taking the inverse of (1) for constant surfaces of $F_{10.7}$ and $h$ respectively. The result is provided in Fig. 1. As pointed out by Pfitzer, the trapped protons have a very slow response time to dynamic changes in atmospheric density $\rho(t)$. Therefore, the values of $F_{10.7}$ and $F_{b a r}$ are assumed identical, whereby $F=F_{10.7}+F_{b a r}=2 F_{10.7}$ in (2b). As stated earlier, the values of $F_{10.7}$ used for solar max and solar min in the following regression analysis become 150 and 70 respectively.

\section{Regression Algorithm}

Several methods and approaches are available to generate a semi-empirical formula for proton flux $J$ as a bivariant function of density $\rho$ (altitude $h$ ) and energy $E$. The method adopted here is taken from theoretical nuclear physics (Lodhi and Waak, $1975,1976)$ based upon a polynomial regression analysis. It is used to determine the functional relationship between fluxes at solar maximum and minimum. Since the proton lifetime $\tau$ is determined by energy losses primarily due to multiple Coulomb scattering from charged constituents in the upper thermosphere, as well as some neutral scattering, it is a function of time $t$, proton energy $E$, and atmospheric density $\rho$ or altitude $h$. That is, $\tau=\tau\left(E, \rho^{-1}, t\right)$ or $\tau=\tau(E, h, t)$ since the atmosphere expands and contracts at different times during the solar cycle.

Utilizing the regression analysis technique, one keeps the regression coefficient greater than $90 \%$. A ratio $J_{\max } / J_{\min }$ or $J_{\min } / J_{\max }$ is generated for differential proton fluxes at solar max and solar min as a function of density (and 1/density) for energies between 
30 and $300 \mathrm{MeV}$ from AP8 model data within 300 to $600 \mathrm{~km}$. Next this ratio is fitted to some polynomial ranging from linear to fifth-order in $\rho$ and $l / \rho$ for proton energies of 30 , $35,40,45,50,60,70,80,90,100,125,150,175,200$, and $300 \mathrm{MeV}$. Note that this ratio is non-linear in $\rho$ or $1 / \rho$. One also finds that polynomials of higher-order than second result in a better fit for given energy than the second-order. However, a common expression for the entire energy range gets worse than that of the second-order. This observation limits the method to be confined to a second-order expression for the flux ratio as a function of $\rho_{\max }$ or $\rho^{-1}$ max for all energies. The regression analysis works well within the energy and altitude range adopted. For other ranges of approximation one has to be careful and do the analysis again, piecewise fitting the entire dynamical range.

\section{Density Dependence}

First the flux ratio is generated as a function of $\rho$ in the quadratic form. The coefficients of $\rho$ vary while progressing from one energy to the next. The flux ratio can then be written in the following fashion:

$$
J_{\min } / J_{\max }=a \rho^{2}+b \rho+c
$$

where $a, b$, and $c$ are proton energy coefficients. Naively, one might hope that these are constant coefficients. However, one discovers that $a, b$, and $c$ are functions of energy $E$ for the 15 different energy values chosen. 
The next step is to find a common expression for this ratio for all energy values. That is achieved by obtaining a functional relation for the coefficients in (3) as a function of energy, again by the method of regression. One obtains four relations for $\rho$ ranging from linear to fourth-order in energy. One also finds that expressions for coefficients $a$, $b$, and $c$ cannot be of the same polynomial order in reproducing the flux ratio. The best fits found for two different energy ranges are as follows:

$$
\begin{aligned}
& J_{\min } / J_{\max }=\left(a_{2} E^{2}+a_{1} E+a_{0}\right)_{l e} \rho^{2}+\left(b_{4} E^{4}+b_{3} E^{3}+b_{2} E^{2}+b_{1} E+b_{0}\right)_{l e} \rho \\
& +\left(c_{4} E^{4}+c_{3} E^{3}+c_{2} E^{2}+c_{1} E+c_{0}\right)_{l e} \quad, \quad 30<E \leq 60 \mathrm{MeV} \\
& J_{\min } / J_{\max }=\left(a_{1} E+a_{0}\right)_{h e} \rho^{2}+\left(b_{1} E+b_{0}\right)_{h e} \rho \\
& +\left(c_{2} E^{2}+c_{1} E+c_{0}\right)_{h e} . \quad 70 \leq E<300 \mathrm{MeV}
\end{aligned}
$$

The coefficients within parentheses are different for le (low energy) and he (high energy). The actual coefficients and a check for the accuracy are given below under Results.

\section{Inverse Density Dependence}

In contrast to (3), the inverse algorithm can be derived. It is known that the atmospheric densities decrease as the altitude increases or the reciprocal of the density increases as the altitude increases. The flux variation with respect to the inverse of the density must convey a direct relation to the variation of the altitude. One must therefore 
search for a similar expression giving the flux ratio as a function of the inverse density. After several trials the best-fitted function thus obtained is given in the form:

$$
\begin{gathered}
J_{\max } / J_{\min }=\left(a_{4} E^{4}+a_{3} E^{3}+a_{2} E^{2}+a_{1} E+a_{0}\right) \rho^{-2}+\left(b_{3} E^{3}+b_{2} E^{2}+b_{1} E\right. \\
\left.+b_{0}\right) \rho^{-1}+\left(c_{2} E^{2}+c_{1} E+c_{0}\right)
\end{gathered}
$$

for all energies $E$ under consideration. This expression is further approximated by writing the coefficients in the exponential form, thus yielding:

$$
J_{\max } / J_{\min }=A e^{\alpha E} \rho^{-2}+B e^{\beta E} \rho^{-1}+C e^{\gamma E}
$$

for all proton energy ranges from 30 to $300 \mathrm{MeV}$.

Results at Solar Max

The two algorithms (3) and (7) are now compared at solar maximum. The resultant semi-empirical formula for the flux ratio in (3) as a function of $\rho$ (in units of $10^{-}$ ${ }^{15} \mathrm{~g} / \mathrm{cm}^{3}$ ) is given by:

$$
\begin{aligned}
& \left(J_{\min } / J_{\text {max }}\right)_{l e}=\left(-3 \times 10^{-8} E^{2}+1 \times 10^{-5} E-8.0 \times 10^{-4}\right) \rho^{2} \\
& \quad+\left(7 \times 10^{-10} E^{4}-5 \times 10^{-7} E^{3}+1 \times 10^{-4} E^{2}-1.4 \times 10^{-2} E+0.695\right) \rho \\
& \quad+\left(-2 \times 10^{-11} E^{4}-5 \times 10^{-8} E^{3}+4 \times 10^{-5} E^{2}-8.6 \times 10^{-3} E+1.897\right)
\end{aligned}
$$




$$
\begin{gathered}
30 \leq E \leq 60 \mathrm{MeV} \\
\left(J_{\text {min }} / J_{\text {max }}\right)_{h e}=\left(2 \times 10^{-6} E+1 \times 10^{-4}\right) \rho^{2}+\left(-7 \times 10^{-4} E+0.181\right) \rho \\
+\left(6 \times 10^{-6} E^{2}-3.7 \times 10^{-3} E+1.66\right) . \\
70 \leq E \leq 300 \mathrm{MeV}
\end{gathered}
$$

To check formulas (8) and (9) for $J_{\min }$, an example at $400 \mathrm{~km}$ and proton energy of $100 \mathrm{MeV}$ is taken, and the results summarized in Table 1. Let us define

$$
f(\rho)=a \rho^{2}+b \rho+c
$$

on the right-hand-side of (3), (8), and (9).

At altitude $400 \mathrm{~km}$ (Heynderickx et al., 2004), the AP8 model in SPENVIS gives

$$
\begin{aligned}
& \rho_{\text {max }}=3.8 \times 10^{-15} \mathrm{~g} / \mathrm{cm}^{3} \\
& \rho_{\text {min }}=9.57 \times 10^{-16} \mathrm{~g} / \mathrm{cm}^{3} \\
& J_{\max }(100 \mathrm{MeV})=2.79 \times 10^{-2} \mathrm{~cm}^{-2} \mathrm{~s}^{-1} \mathrm{MeV}^{-1} \text { (SPENVIS) }
\end{aligned}
$$

For this density at solar max one obtains $f\left(\rho_{\max }\right)=f(3.8)$ in $(10)$ and $J_{\min } / J_{\max }=1.78$ in (3). It then follows from (3) that

$$
J_{\min }{ }^{\left({ }^{(A g o r i t h m}\right)}=J_{\max }{ }^{(\operatorname{SPENVIS})} f\left(\rho_{\max }\right)=\left(2.79 \times 10^{-2} \mathrm{~cm}^{-2} \mathrm{~s}^{-1} \mathrm{MeV}^{-1}\right)(1.78)
$$


On the other hand, AP8 (Heynderickx et al., 2004) gives

$$
J_{\min }{ }^{(\text {SPENVIS })}=5.151 \times 10^{-2} \mathrm{~cm}^{-2} \mathrm{~s}^{-1} \mathrm{MeV}^{-1}
$$

Comparison of (11) and (12) yields a difference of $0.183 \times 10^{-2} \mathrm{~cm}^{-2} \mathrm{~s}^{-1} \mathrm{MeV}^{-1}$ with an error of $3.5 \%$. These are summarized in Table 1.

Next, the resultant semi-empirical formula for the flux ratio $J_{\max } / J_{\min }$ in (7) as a function of $1 / \rho$ (in units of $10^{+15} \mathrm{~cm}^{3} / \mathrm{g}$ ) is determined by regression analysis to have the form:

$$
\begin{gathered}
J_{\max } / J_{\min }=-0.0241 e^{0.0007 E} \rho^{-2}+0.1966 e^{-0.0007 E} \rho^{-1} \\
+0.3208 e^{+0.0032 E}
\end{gathered}
$$

for all energies between 30 and $300 \mathrm{MeV}$.

Following the same procedure used in (10) and (11), we can define

$$
g\left(\rho^{-1}\right)=A e^{\alpha E} \rho^{-2}+B e^{\beta E} \rho^{-1}+C e^{\gamma E}
$$


for the right-hand-side of (7), (8), (9), and (13). One determines that $g\left(\rho^{-1}{ }_{\max }\right)=0.4912$ in (14). The resultant $J_{\min }$, the difference from SPENVIS, and the error are summarized in Table 1.

For further comparison, from expression (13) for $J_{\max }\left(\mathrm{cm}^{-1} \mathrm{~s}^{-1} \mathrm{MeV}^{-1}\right)$ a differential flux is calculated and contrasted with the AP8 data in Fig. 2, derived from SPENVIS (Heynderickx et al., 2004) for an ISS orbit of 350-478 km altitude and inclination $51.65^{\circ}$. Fig. 2 is a two-dimensional projection of the three-dimensional surface $J(h, E)$ at various selected altitude $h$. The NOAAPRO results in SIREST are shown in Fig. 3 and Fig. 4 along with the algorithm at $400 \mathrm{~km}$ and $500 \mathrm{~km}$ altitudes, for solar max with $F_{10.7}=150 \mathrm{in}$ the algorithm.

Results Midway between Solar Max and Solar Min

Method 1. By varying the solar-cycle modulation parameter $M$ in (2), one obtains a different atmospheric density model in (1). This can be accomplished by changing $F_{10.7}$ and $F_{b a r}$ whereby a different value of density $\rho$ is obtained, either from (1) or from the carpet plot in Fig. 1. Upon insertion of the new value of density $\rho$, a proton differential flux follows from (3) and (7). The baseline regression algorithms (3) and (7) assume $F_{10.7}=70$ and $F_{10.7}=150$ in Fig. 3 and Fig. 4 for solar min and max respectively. In order to determine the proton differential flux mid-way through this same adopted cycle, the solar flux becomes $F_{10.7}=110$ whereby $F=220$ in (2b). The resulting proton differential spectrum is shown in Fig. 5 for $400 \mathrm{~km}$ in Fig. 6 for $500 \mathrm{~km}$. 
Method 2. Given a proton flux $J$ at either solar maximum or minimum, such as the algorithm in (3) and (7), then an interim flux is determinable as a linear timeinterpolation,

$$
J(E, h, \tau) \sim J_{\max }(E, h, \tau)(1-\Gamma)+\Gamma J_{\min }(E, h, \tau),
$$

or alternatively,

$$
J(E, h, \tau) \sim J_{\min }(E, h, \tau)(1-\Gamma)+\Gamma J_{\max }(E, h, \tau)
$$

where $\Gamma(E, \rho, \tau)$ is the dimensionless fraction

$$
\Gamma(E, \rho, \tau)=\frac{\tau-\tau_{\max }(E)}{\tau_{\min }(E)-\tau_{\max }(E)}
$$

The lifetime $\tau$ is assumed to be limited to one solar cycle or 11 years.

To calculate the desired intermediate proton flux $J(E, h, \tau)$ at a time between solar maximum and solar minimum using Method 2, the right-hand-side of (17) represents the interpolation fraction $\Gamma$ of the solar cycle since last solar minimum. Then either of (15) and (16) gives the interim flux in this approximation. Substituting (3) and (7) into (15) and (16) respectively, one has 


$$
\begin{aligned}
& J(E, h, \tau) \sim J_{\max }(E, h, \tau)\left\lfloor(1-\Gamma)+\Gamma\left(a \rho^{2}+b \rho+c\right)\right\rfloor, \\
& J(E, h, \tau) \sim J_{\min }(E, h, \tau)\left\lfloor(1-\Gamma)+\Gamma\left(A e^{\alpha E} \rho^{-2}+B e^{\beta E} \rho^{-1}+C e^{\gamma E}\right)\right\rfloor .
\end{aligned}
$$

The various coefficients in (18) and (19) are given in (4)-(5) and (8)-(9). Further study is planned to conduct an error analysis between Method 1 and Method 2.

\section{Conclusions}

The proton differential flux has been expressed, empirically, as a bivariant function of density (altitude) and energy, broken into two ranges of proton energy, viz., 30 to $60 \mathrm{MeV}$ and 70 to $300 \mathrm{MeV}$. The corresponding expression in terms of inverse density is relatively compact and works for the entire range of proton energy, 30 to 300 $\mathrm{MeV}$. From this analysis it is observed that the proton differential flux has a nonlinear dependence on energy and density (altitude). The flux ratio has been expressed in a semi-empirical form given by (3) and (7). It has been compared with AP8 model data as shown in Fig. 2 for Shuttle and ISS altitudes of current interest. An additional comparison with NOAAPRO is given in Fig. 3 and Fig. 4. An illustration of the algorithm mid-way through the adopted solar cycle is produced in Fig. 5 and Fig. 6. Finally, the algorithm provides a simple means for calculating the trapped-belt proton flux when the $F_{10.7}$ solar modulation flux is 200 or greater. The analysis thus avails itself to other more prominent solar sycles where AP8 is not valid. However, a thorough error analysis will be required in the future in order to determine the general limitations of this 
regression-analysis algorithm. As a concluding remark, the selection of solar flux $F_{10.7}$ is a matter of convention due to its known correlation with sunspot number. The physics of the thermosphere is not completely understood and there is current interest in the extreme ultraviolet parameter $E_{10.7}$. Should another modulation factor be found, the regression analysis presented here can be modified to accommodate it.

\section{Acknowledgement}

Two of us (M.A.K.L. and A.B.D.) would like to thank Dr. Gautam Badhwar (deceased) for suggesting that the Pfitzer density-dose model has merit and should be investigated further for flux. This was during the Summer Faculty Fellowship (SFF) program at the Johnson Space Center, Houston, Texas, in 2001, when the initial phase of this investigation began. All of us also thank Dr. Stuart Huston for valuable comments regarding NOAAPRO as well as an anonymous reviewer for pointing out its relevance to this analysis. Finally we are grateful to Dr. Karl Pfitzer for providing details of his model. This regression analysis was completed during a second invitation to JSC in 2004 for the SFF program there. 


\section{References}

Badhwar, G.D., 1999. Radiation dose rates in Space Shuttle as a function of atmospheric density, Rad. Meas. 30, 401-414.

Badhwar, G.D., Shurshakov, V.A., and V.V. Tsetlin, V.V., 1997. Solar Modulation of

Dose Rate Onboard the Mir Station, IEEE Trans. on Nuclear Science 44, no. $6,2529-2541$.

Badhwar, G.D., Konradi, A., Atwell, W., Golightly, M.J., Cucinotta, F.A., Wilson, J.W., Petrov, V.M., Tchernykh, I.V., Shurshakov, V.A., and Labokov, A.P., 1996a. Measurements of the linear energy transfer spectra on the Mir orbital station and comparison with radiation transport models, Rad. Meas. 26, 147-158.

Badhwar, G.D., Golightly, M.J., Konradi, A., Atwell, W., Kern, J.W., Cash, B., Benton, E.V., Frank, A.L., Sanner, D., Keegan, R.P., Frigo, L.A., Petrov, V.M., Tchernykh, I.V., Akatov, Y.A., Shurshakov, V.A., Arkhangelsky, V.V., Kushin, V.V., Klychin, N.A., Vana, N., and Schoner, W., 1996b. In-flight radiation measurements on STS-60, Rad. Meas. 26, 17-34.

Bilitza, D., 1987. Models of the trapped particle fluxes AE-8 (electrons) and AP8 (protons) in inner and outer radiation belts, Nat. Spa. Sci. Data Center, Goddard Space Flight Center, NSSDC, October.

Blanchard, R.C., and Hess, W.N., 1964. Solar cycle changes in inner-zone protons, J. Geophys. Res. 69, 3927-3938. 
Cornwell, J.M., Simms, A.R., and White, R.S., 1965. Atmospheric density experienced by radiation belt protons, J. Geophys. Res. 70, 3099-3111.

Dragt, A.J., 1966. Solar cycle modulation of the radiation belt proton flux, J. Geophys. Res. 76, 2313-2344.

Golightly, M.J., Badhwar, G.D., Dunlap, M.J., Patel, S.H., 1996. Solar-Cycle modulation of the trapped proton radiation exposure inside the Space Shuttle. In: Balasubramaniam, K.S., Keil, S.L., and R.N. Smartt, R.N. (Eds.), Solar Drivers of the Interplanetary and Terrestrial Disturbances, Vol. 95, Astronomical Society of the Pacific Conference Series, 505-517.

Harris, I., and Priester, W., 1962. Theoretical models for the solar-cycle variation of the upper atmosphere, J. Geophys. Res. 67, 4585-4591.

Harris, I., and Priester, W., 1963. Relation between theoretical and observational models of the upper atmosphere, J. Geophys. Res. 68, 5891.

Heynderickx, D., et al., 2004. SPace ENVironment Information System (SPENVIS), European Space Agency (ESA), http://www.spenvis.oma.be/spenvis/ .

Huston, S.L., and Pfitzer, K.A., 1998a. A new model for the low altitude trapped proton environment, IEEE Trans. On Nuclear Science 45, no. 6, 2972-2978.

Huston, S.L., and Pfitzer, K.A., 1998b. Space environment effects: Low-altitude trapped radiation models, Marshall Space Flight Center, NASA/CR-1998-208593, 63 pp., available at http://see.msfc.nasa.gov/ire/irepub.html.

Jacchia, L.G., 1960. A variable atmospheric-density model from satellite accelerations, J. Geophys. Res. 65, 2775-2782. 
Jacchia, L.G., 1961. A working model for the upper atmosphere, Nature 192, 1147-1148.

Kern, J.W., 1994. A note on vector flux models for radiation dose calculations, Rad.

Meas. 23, 43-48.

Lodhi, M.A.K., and Waak, B.T., 1975. Solution of bound state problems in nuclear shell models with momentum-dependent potentials, Comm. Phys. Comm. 10, 182-193.

Lodhi, M.A.K., and Waak, B.T., 1976. A Momentum-Dependent Potential Approximated by the Morse Function for Studying Nuclear Systematics, Ann. Phys. $101,1-21$.

Pfitzer, K.A., 1989. Space station radiation dose as a function of atmospheric density, McDonnell Douglas Space Syst. Co., Advanced Technology Center, Huntington Beach, CA 92647, MDSSC Rep. no. H5387.

Ray, E.C., 1966. On the theory of protons trapped in the Earth's magnetic field, J. Geophys. Res. 65, 1125-1134.

Sawyer, D.M., and Vette, J.I., 1976. AP8 trapped proton environment for solar maximum and solar minimum, Nat. Spa. Sci. Data Center, Goddard Space Flight Center, NSSDC/WDC-A-R\&S 76-06.

Schulz, M. and Lanzerotti, L.J., 1974. Particle Diffusion in the Radiation Belts, Springer-Verlag, New York.

Singleterry, R., et al., 2004. Space Ionizing Radiation Environments and Shielding Tools (SIREST), NASA, http://sirest.larc.nasa.gov/. 
Spejeldvik, W.N. and P.L. Rothwell, 1985. The Radiation Belts. In: Handbook of Geophysics and the Space Environment, Jursa, A.S. (Ed.), AFGL, USAF, DA167000, 5-1.

Watts, J.W., Parnell, T.A., and Heckman, H.H., 1989. Approximate angular distribution and spectra for geomagnetically trapped protons in low-Earth orbit. In: HighEnergy Radiation Background in Space, Rester, A.C., Jr., and Trombka, J.I. (Eds.), AIP Conf. Proc. 186, New York, 75-85. 


\section{Figure 1 Caption}

Carpet plot of the solar-cycle terms altitude $h$ and modulation flux $F_{10.7}$, as a function of atmospheric density in Equations (1) and (2).

\section{Figure 2 Caption}

Graph of proton differential flux versus energy at various Shuttle and International Space Station altitudes, comparing the present algorithm with predictions of AP8 at solar maximum.

\section{Figure 3 Caption}

Graph of proton differential flux versus energy at $400 \mathrm{~km}$ altitude. Proton flux models AP8MAX, SIREST/NOAAPRO, and the algorithm presented here are compared at solar maximum with $F_{10.7}=150$. AP8MIN is also given.

\section{Figure 4 Caption}

Graph of proton differential flux versus energy, like Figure 3, except at $500 \mathrm{~km}$ altitude. Proton flux models SIREST/NOAAPRO, and the algorithm presented here are compared at solar maximum with $F_{10.7}=150$. AP8MAX is not illustrated since it is essentially identical to SIREST at this altitude. AP8MIN is also given. 


\section{Figure 5 Caption}

The same graph as in Figure 3, except with the algorithm initialized for half-way through the assumed solar cycle assuming $F_{10.7}=F_{b a r}=110$ in Eq. (1) and (2).

\section{Figure 6 Caption}

The same graph as in Figure 4, except with the algorithm initialized for half-way through the assumed solar cycle assuming $F_{10.7}=F_{b a r}=110$ in Eq. (1) and (2). 


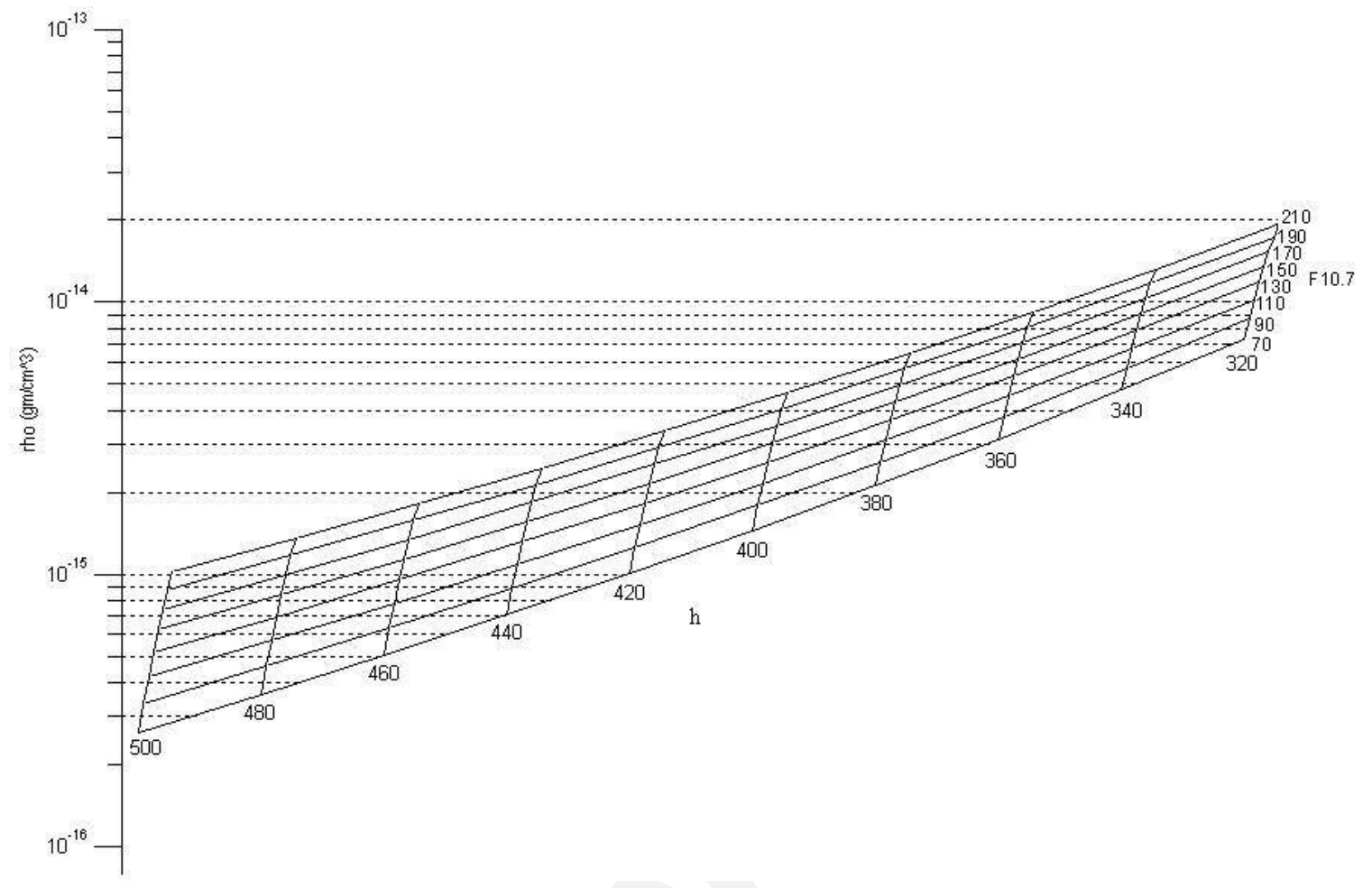

Figure 1 


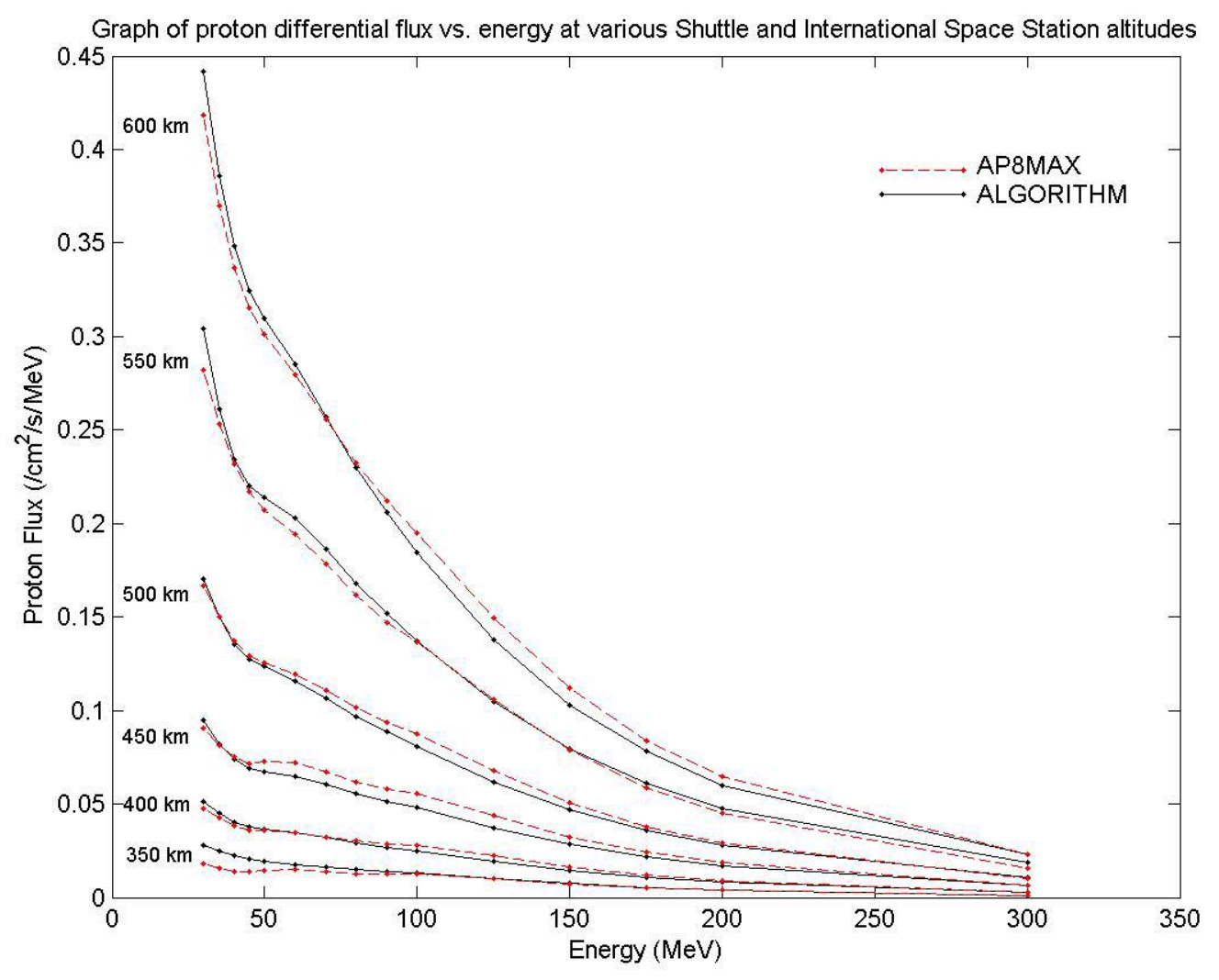

Figure 2 


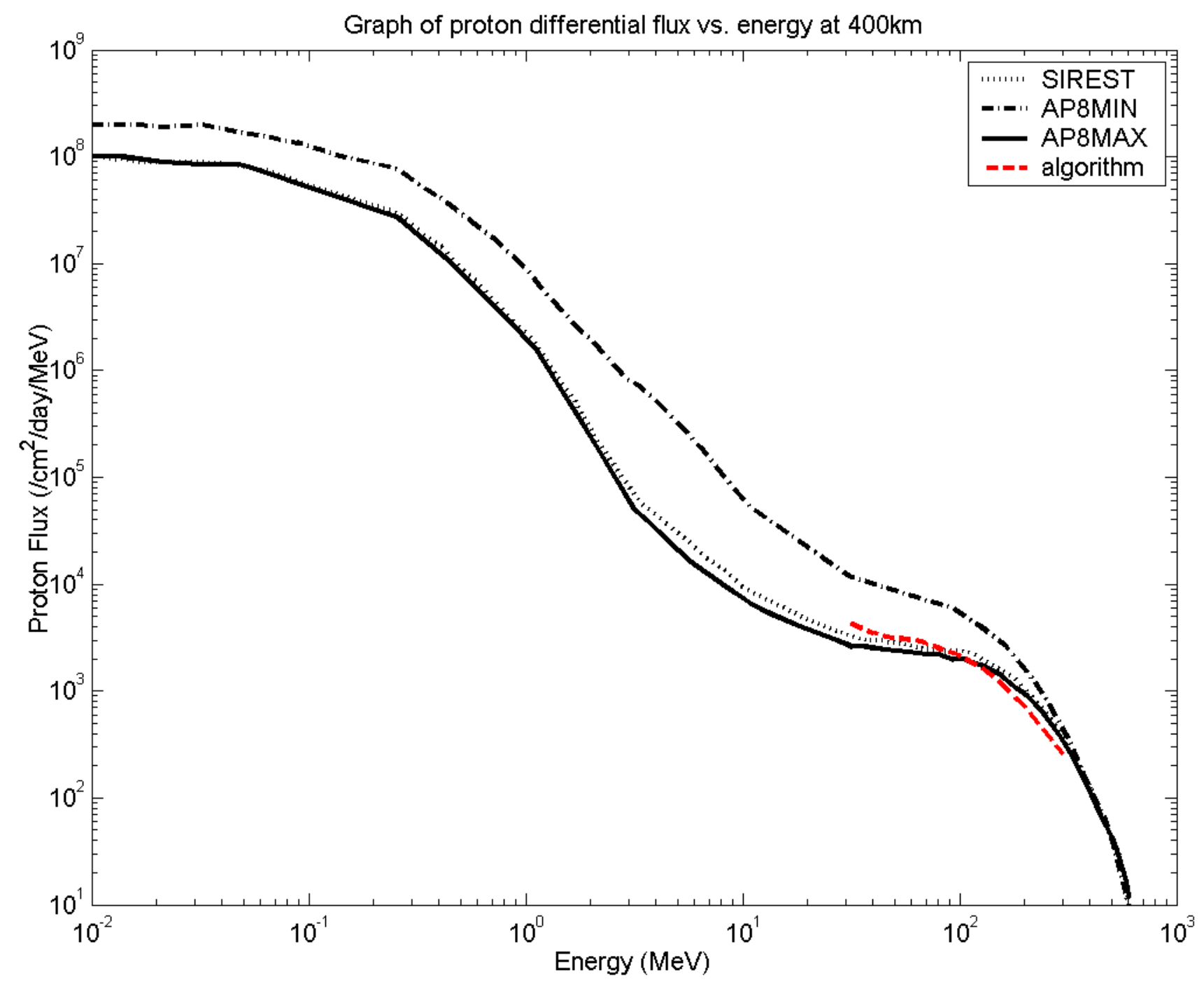

Figure 3 


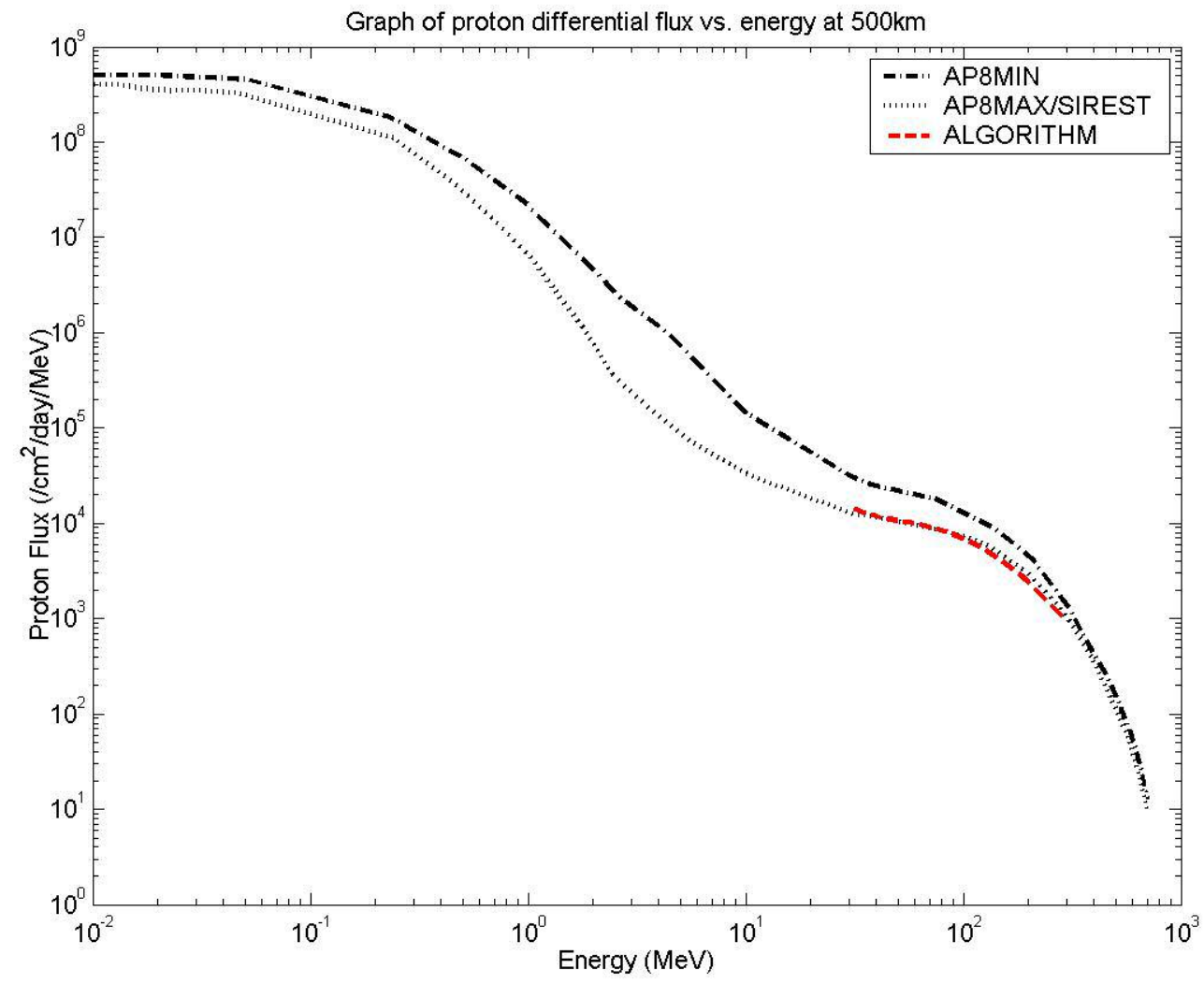

Figure 4 


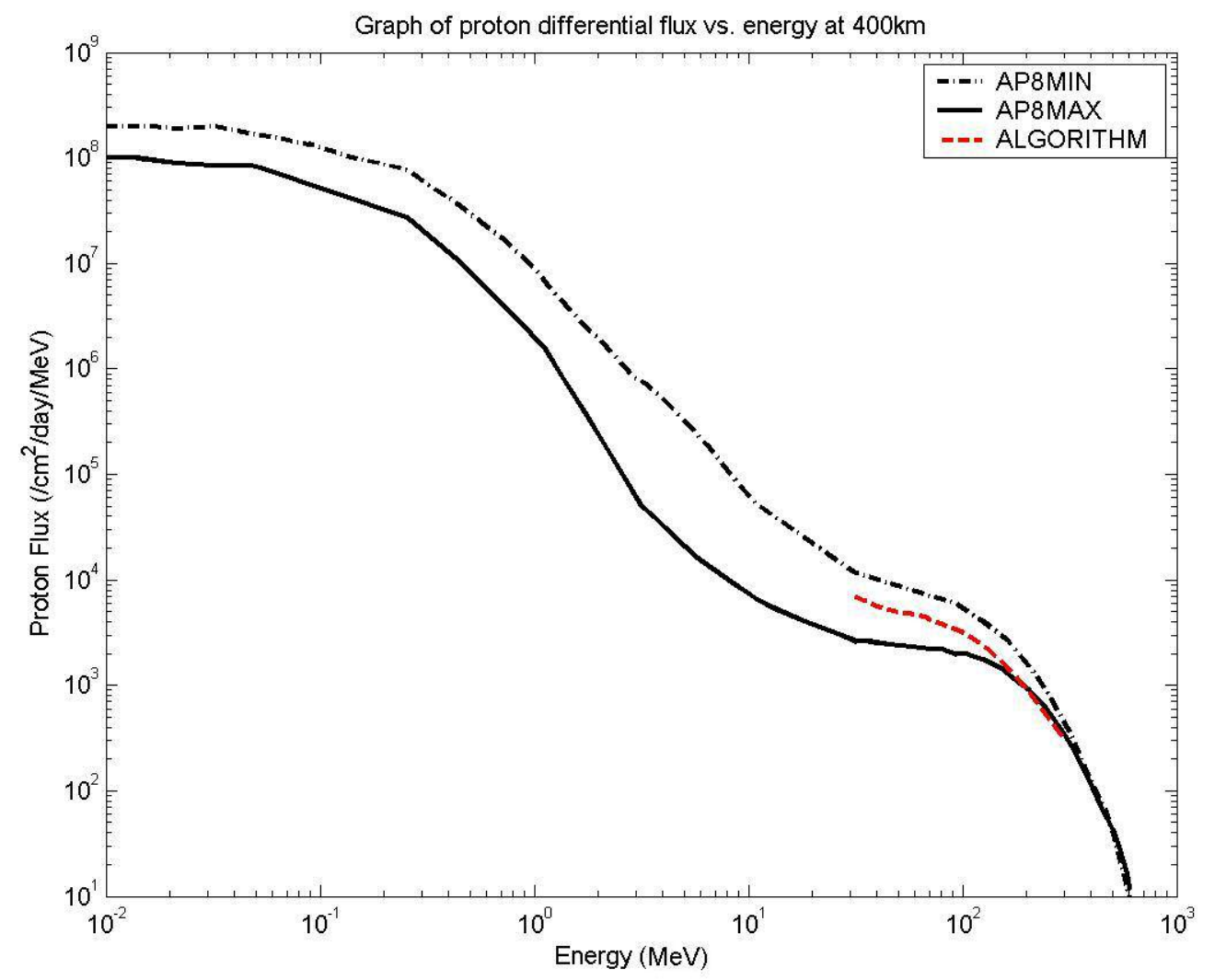

Figure 5 


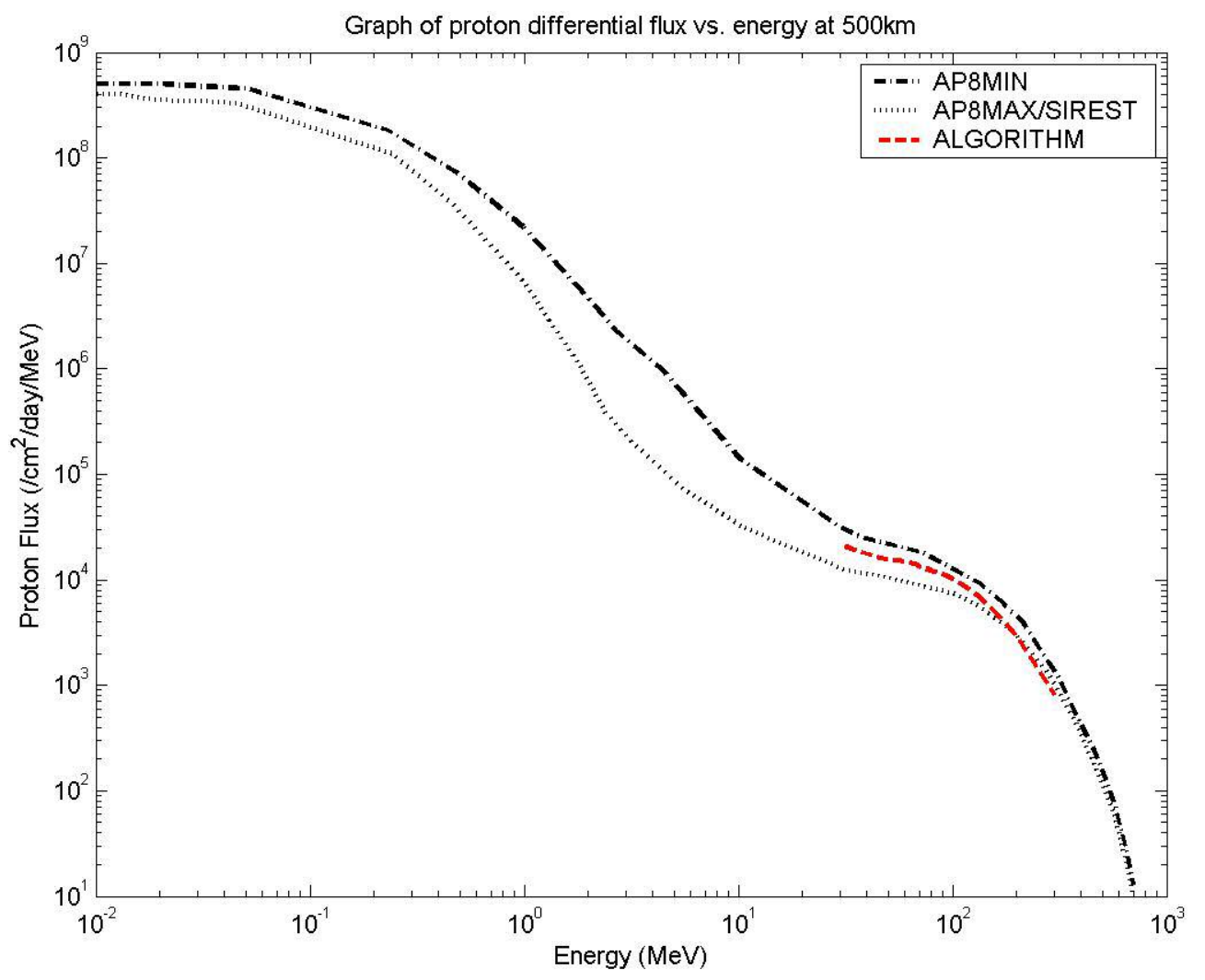

Figure 6 


\section{Table and Table Caption}

Table 1

Comparison of $J_{\text {min }}{ }^{(\text {Algorithm })}$ with $J_{\min }{ }^{(S P E N V I S)}$ for proton energy of $100 \mathrm{MeV}$ at $400 \mathrm{~km}$.

\begin{tabular}{|c|c|c|}
\hline SPENVIS Parameter & Value & Value \\
\hline$\rho_{\max }$ & $3.8 \times 10^{-15}$ & \\
\hline$\rho_{\min }$ & $9.57 \times 10^{-16}$ & \\
\hline$J_{\max }^{(S P E N V I S)}$ & $2.79 \times 10^{-2}$ & \\
\hline$J_{\min }^{(S P E N V I S)}$ & $5.15 \times 10^{-2}$ & \\
\hline$J_{\min }{ }^{(S P E N V I S)} / J_{\max }{ }^{(S P E N V I S)}$ & 1.78 & \\
\hline Comparison with Algorithm & Algorithm (3) & Algorithm (7) \\
\hline$J_{\text {min }}{ }^{(\text {Algorithm })}$ & 4.955 & 5.680 \\
\hline Difference from $J_{\min }{ }^{\text {(SPENVIS) }}$ & 0.183 & 0.53 \\
\hline$\%$ Error & 3.5 & 10.3 \\
\hline
\end{tabular}

Units of $\rho$ are in $\mathrm{g} / \mathrm{cm}^{3}$ and those of $J$ are $\mathrm{cm}^{-2} \mathrm{~s}^{-1} \mathrm{MeV}^{-1}$. 